\title{
Numerical Elucidation of Flow and Dispersion in Ordered Packed Beds: Non-Spherical Polygons and the Effect of Particle Overlap on Chromatographic Performance
}

Fabian Dolamore $^{1 *}$, Simone Dimartino ${ }^{2}$ and Conan J. Fee ${ }^{1,3}$

${ }^{1}$ Biomolecular Interaction Centre, ${ }^{3}$ School of Product Design, University of Canterbury, Private Bag 4800, Christchurch, New Zealand 8041

${ }^{2}$ Institute for Bioengineering, School of Engineering, The University of Edinburgh, Edinburgh EH9 $3 \mathrm{FB}, \mathrm{UK}$

Table of Contents

1. Discussion on the characteristic length scale of a porous medium

2. Particle rotation - A fifth morphological variable

3. Supplementary data sets to the main text

\section{Corresponding Author}

Fabian Dolamore

*E-mail: Fabian.dolamore@ canterbury.ac.nz

Phone: +6427 7282030 


\section{S1. Characteristic Length Scale}

Here, we provide a justification on the choice of our characteristic length scale as mentioned in Section 3.2 of the main work.

In physical systems, a characteristic length represents a reference scale upon which important phenomena occur. Selection of an appropriate characteristic length is crucial because it dictates how we compare results between different systems. More specifically, in this work $d$ is used to calculate $D a(\mathrm{Eq})$ and $h(\mathrm{Eq})$, two important chromatographic performance metrics. The problem of finding a suitable characteristic length has been discussed by De Malsche et al. when attempting to compare results between $2 \mathrm{D}$ pillar arrays and randomly packed beds ${ }^{1}$. It was suggested that the pore width is roughly $1 / 3$ of the particle diameter in these systems, however these types of heuristic are not applicable when dealing with ordered packings and non-spherical particle shapes.

In mono-disperse randomly packed beds of spheres, the particle diameter is often used as the characteristic length, because it is a well-defined and convenient quantity. Determining alternatives for random structures, such as pore diameter, is a non-trivial exercise, as has been discussed by Guiochon in the context of monoliths ${ }^{2}$. However, the concept of particle diameter is lost for morphologies composed of non-spherical particles and monoliths, hence a robust alternative is now required.

Because additive manufacturing affords us with much fine control over our bed structure, we can predetermine and characterise the pore structure of these media using computational tools. Here, we discuss several potential definitions of characteristic length.

\section{S1.1 Characteristic Particle Length $\left(d_{p}\right)$}

A characteristic length related to some fundamental particle dimension has been the preferred definition used in the literature related to packed beds of spherical particles ${ }^{3-6}$. In the context of random beds of discrete, mono-disperse spherical particles, the characteristic length is simply the diameter of the packed spheres, or an average diameter for poly-disperse or non-spherical particles ${ }^{7}$.

Bead diameter is a description of the stationary phase, whereas flow and dispersion occur in the mobile phase. While the use of bead diameter does not seem to be appropriate in principle, all random packings are assumed to be geometrically similar, including the resulting network of channels. Thus, particle diameter is uniquely related to a certain characteristic dimension describing the porous channel network and the associated flow of the mobile phase and the two characteristic lengths for the solid and mobile phases are employed interchangeably. However, this becomes invalid when considering regular packing of spheres as the channels formed strongly depend on the configuration (e.g. SC, BCC or FCC) of the ordered bed.

A corresponding parameter for non-spherical particles may be the equivalent diameter (i.e. the diameter of a sphere with equivalent volume) or another canonical dimension of the particle (e.g. the circumdiameter, middiameter or indiameter for the two Platonic solids considered in this work). Yet, as already mentioned for regular packing of spheres, a characteristic dimension for the particles does not directly reflect the shape and size of the channels formed, especially when configuration is considered, and thus it is not entirely representative for physical phenomena occurring in the mobile phase.

It should be noted that if band-broadening within the stationary phase were to be considered (i.e. a porous solid-phase material), the characteristic dimension of the stationary phase would become important. However, in this work, we neglect solute dynamics within the stationary phase and leave such considerations to future studies.

\section{S1.2 Axial Periodic Length $\left(l_{z}\right)$}

One defining feature of ordered packed beds is the unit cell, which has the property of regular three-dimensional periodicity. The unit cell allows us to define the smallest repeating unit of an ordered packing, potentially making it an attractive choice for comparing packings that are required to occupy a defined physical space, e.g. in a chromatography column.

Using the example of sphere packings, SC, BCC and FCC unit cells containing spheres of identical size would not have the same axial length: a given number of FCC unit cells would extend over a greater length than the same number of SC or BCC cells. Thus, a column of equal length may have less resolving power in practice because the mobile phase has encountered fewer unit cells. Using the axial unit cell length as the non-dimensionalizing factor addresses this concern because the normalization asserts that all unit cells are of equivalent length.

However, this normalization also alters the particle size, i.e. unit cells with larger axial lengths would be normalized to a smaller particle size. Because it is known that particle and channel size directly influence mobile phase band broadening effects ${ }^{8}$, this method would give the illusion of better performance for longer unit cells. Furthermore, this method is not universally applicable. For example, there are no repeating unit cells in randomly packed beds and instead the particle diameter is commonly used ${ }^{5,9}$. There are also special cases of monolithic media, such as straight channels where a periodic length cannot be defined. Thus, unit cell (axial) length does not appear to be a useful choice of characteristic length for analytical purposes. 


\section{S1.3 Hydraulic Diameter $\left(d_{h}\right)$}

The characteristic length often employed in channel flow is the hydraulic diameter, $d_{h}[\mathrm{~L}]$, i.e. the ratio between the channel area cross-sectional $A\left[\mathrm{~L}^{2}\right]$ and the wetted perimeter $P[\mathrm{~L}]$. However, the channels through packed beds (either ordered or random) typically do not have a uniform profile along the axial dimension, and the definition of hydraulic diameter is conveniently extended into a three dimensional approach ${ }^{10}$.

$$
d_{h}=\left[\frac{4 A}{P}\right]_{2 D}=\left[\frac{4 \varepsilon}{a}\right]_{3 D}
$$

where $a$ is the specific packing area $\left[\mathrm{L}^{-1}\right]$, i.e. the surface area per unit volume of the packed bed. When reporting $d_{h}$ values, we assume that all particles in this work have a uniform diameter of one unit, as encompassed by the cells illustrated in Fig. 1 of the main text. By this definition, $d_{h}$ is independent of bed orientation.

\section{S1.4 Comparison of Characteristic Lengths}

The different expressions of characteristic length were employed to describe results reported in the literature for ordered beds of spheres ${ }^{3,6,11}$. In this section, the characteristic length is employed to normalize $P e, D a$ and $h^{12}$. This analysis shows that the apparent hierarchy of relative dispersion performances for SC, BCC and FCC packings in van Deemter plots depends critically upon the characteristic length definition (Fig. S1). If hydraulic diameter is used to calculate the characteristic length (triangles), BCC packing has the worst performance, contrary to the traditional literature based upon (equivalent) particle diameter, in which SC is reported to have the worst performance. The performance hierarchy remains unchanged when comparing $d_{p}$ and $l_{z}$.

While the definition of characteristic length changes $D a$ and $h$, it has no effect on $E$. This can be seen by substituting Eqs. 7 and 8 into Eq. 9 (Table S1). It is also worth noting that there is agreement between the hierarchy of $h$ and $E$ for these media when using $d_{h}$ as the characteristic length, despite the independence of $E$ from characteristic length. It is also interesting to note that, when using $d_{p}$ to calculate $D a$, they become of equivalent order of magnitude, which is not true for $d_{p}$ or $l_{z}$.

It could be argued that any one of these data sets is correct within the rationale used to choose the corresponding characteristic length. If a consistent measure is used, and geometric similarity applies (i.e. spherical beads or non-spherical particles that lend themselves to approximation as "equivalent spheres") one might remain satisfied with use of a characteristic particle dimension such as (equivalent) particle diameter to compare flow characteristics. However, moving forward in the context of 3D-printed porous media, in which "particle" shape, size and alignment as well as packing arrangement and orientation can be controlled and thus mobile phase channel geometries designed and/or defined, it is logical in our view that we must move from considering a particle-based definition to a channel-based definition when defining a characteristic length to compare mobile-phase dispersion. Hence, from this point on, characteristic length refers to the hydraulic diameter, unless otherwise stated.

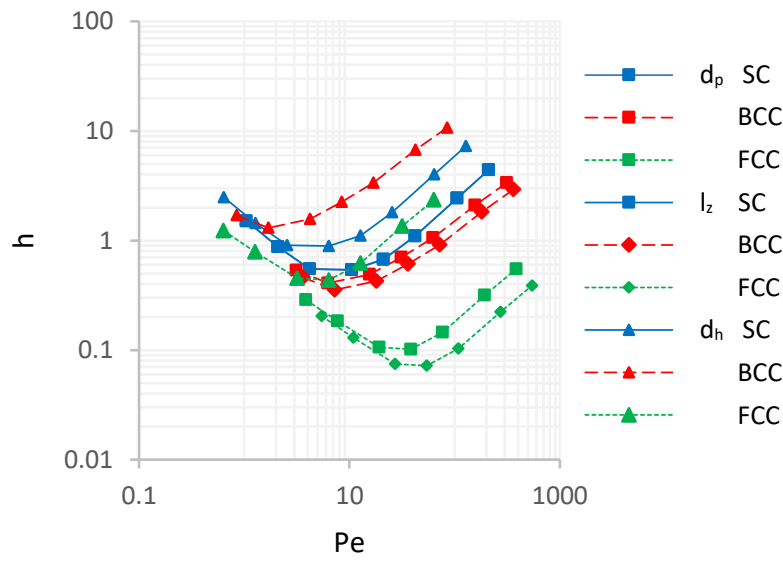

Figure S1. Comparison of van Deemter curves for: SC, BCC and FCC packing of monodisperse spheres using each definition of characteristic length. Note, $d_{p} S C$ and $l_{z} S C$ share identical data points.
Table S1. Performance metrics of different ordered packings of monodisperse spheres obtained using $d_{p}, l_{z}$ and $d_{h}$ as the three different characteristic lengths (denoted as $d$ ).

\begin{tabular}{c|ccccc} 
Method & Config. & $d$ & $D a \times 10^{-3}$ & $h_{\min }$ & $E$ \\
\hline $\mathrm{d}_{\mathrm{p}}$ & SC & 1 & 2.6 & 0.54 & 53 \\
& BCC & 1 & 0.50 & 0.41 & 106 \\
& FCC & 1 & 0.17 & 0.10 & 16 \\
$\mathrm{l}_{\mathrm{z}}$ & SC & 1 & 2.6 & 0.54 & 53 \\
& BCC & 1.15 & 0.38 & 0.35 & 106 \\
& FCC & 1.41 & 0.09 & 0.07 & 16 \\
$\mathrm{~d}_{\mathrm{h}}$ & SC & 0.61 & 7.2 & 0.89 & 53 \\
& BCC & 0.32 & 5.1 & 1.30 & 106 \\
& FCC & 0.23 & 3.2 & 0.44 & 16
\end{tabular}




\section{S2. Particle Rotation}

Particle rotation was not found to improve chromatographic performance of packed beds, thus is presented here. Rotation is denoted as a subscript after the configuration value in our notation, e.g. $\mathrm{SC}_{2}$ [001] $\mathrm{S}_{\lambda=1.00}$ specifies a rotation state of "2", as described below.

Particle rotation is a novel concept that is not relevant in the context of spherical particle packings, because spherical particles are isotropic. For alternative particle shapes, however, the particle's alignment within the unit cell has a pronounced effect on the morphology of the packed bed. There are an infinite number of possible alignments of particles, so in this work, we only consider uniform particle alignments that create a cubic unit cell. For octahedra, there are only two distinct rotations that satisfy this criterion. The first has its vertices on the centre of each face of the unit cell, while the second maximizes the volume of the octahedron within the unit cell (Fig. S2a). For octahedral particles in the second alignment, the particle size must be increased to achieve a continuous bed structure.

For tetrahedra, there is only one rotation that satisfies the cubic unit cell condition if only single particles are considered. To create a second alignment that satisfies a cubic unit cell, multiple particles were packed into the unit cell, creating a tri-tetrahedral structure (Fig. S2b). It was found that tri-tetrahedral packings do not lend themselves to either BCC or FCC arrangements because six of the eight vertexes of the unit cell are occupied, creating large void spaces in these for these configurations, which we know are characteristic of poor performance.

a)

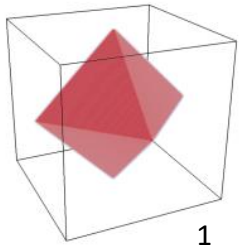

b)

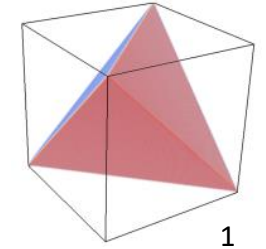

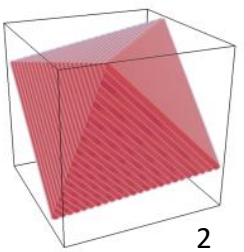

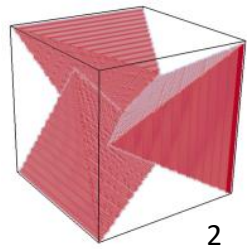

Figure S2. Illustration of a) octahedral and b) tetrahedral rotations studied in this work. Only rotations that created a cubic unit cell in the SC[001] configuration were considered.

Because rotation changes how a particle fits within the unit cell, it can affect the bed porosity, e.g. $\mathrm{SC}_{1}[001]$ octahedra has $\varepsilon=$ 0.82 , while for $\mathrm{SC}_{2}[001] \varepsilon=0.20$. (Table S2). It should be noted that the sphericity of $\mathrm{SC}_{2}$ octahedra is $>1$, because this packing configuration asserts that particles contact along adjacent particle faces, thus reducing the wetted surface area and increasing $\psi$. This geometric interaction is also true for $\mathrm{BCC}_{2}$ octahedra, which exhibits greater sphericity than as a discrete particle.

Rotation of non-spherical particles has a marked impact on mobile phase band broadening, which is independent of the other configuration variables (orientation and arrangement) (Fig. S3). Although some of these media did exhibit low levels of band broadening, they did not challenge the superior performance of the $\mathrm{SC}_{1}$ [111] octahedral bed. It is interesting to note that $D a$ values of the BCC O packings are unaffected by rotation, which supplements our previous observation that pressure drop is independent of orientation, though this is not the case for SC octahedra because the low porosity of the $\mathrm{SC}_{2}$ bed. This not the case for the tetrahedral alignments because the number of particles in the unit cell is increased, which is the same observed behaviour when comparing SC, BCC, and FCC configurations.

These results lead us to conclude that simply manipulating bed and particle features is not an effective way to optimize an ordered medium (when considering mobile phase band broadening), because these variables do not directly control the characteristics of the fluid channels. Instead, the focus in these ordered packed beds should be shifted toward designing the channels, i.e. designing monolithic media as opposed to packed beds. This paradigm shift would allow the manufacturer to optimize the performance of a given medium by identifying and reducing channel features that promote dispersion. 


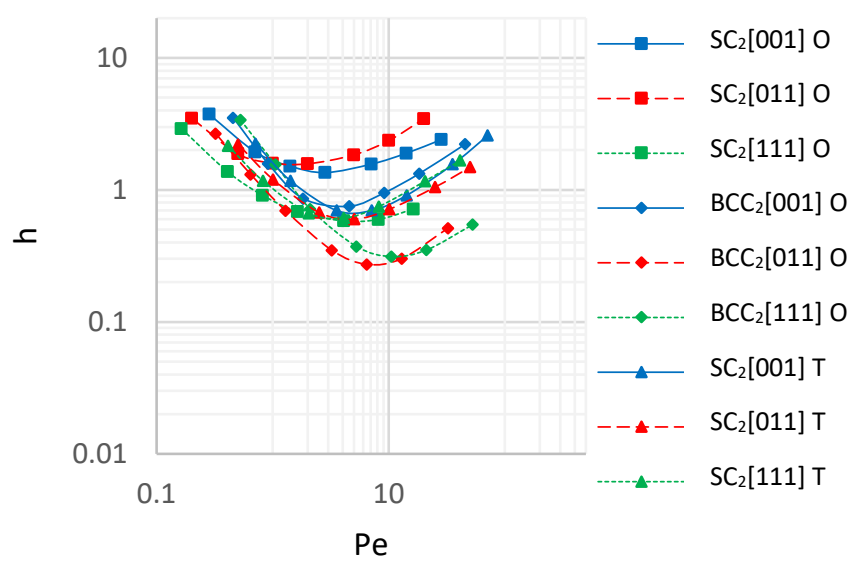

Figure S3. van Deemter curves for the best performing rotation of octahedral and tetrahedral particles as illustrated in Fig. S2.
Table S2. Flow characteristics for alternative alignments of octahedral and tetrahedral particles.

\begin{tabular}{c|cccc} 
Shape & Arrangement & $d_{h}$ & $\varepsilon$ & Da $\times 10^{-3}$ \\
\hline $\mathrm{O}$ & $\mathrm{SC}_{1}$ & 1.92 & 0.82 & 5.8 \\
& $\mathrm{SC}_{2}$ & 0.29 & 0.20 & 1.6 \\
& & & & \\
& $\mathrm{BCC}_{1}$ & 0.66 & 0.62 & 6.8 \\
& $\mathrm{BCC}_{2}$ & 0.50 & 0.47 & 6.7 \\
$\mathrm{~T}$ & & & & \\
& $\mathrm{SC}_{1}$ & 0.77 & 0.66 & 5.6 \\
& $\mathrm{SC}_{2}$ & 0.46 & 0.64 & 8.4
\end{tabular}




\section{S3. Supplementary Figures}

Each of the following figures is an extended data set from the corresponding figures of the main work, as specified in the respective captions.

a)

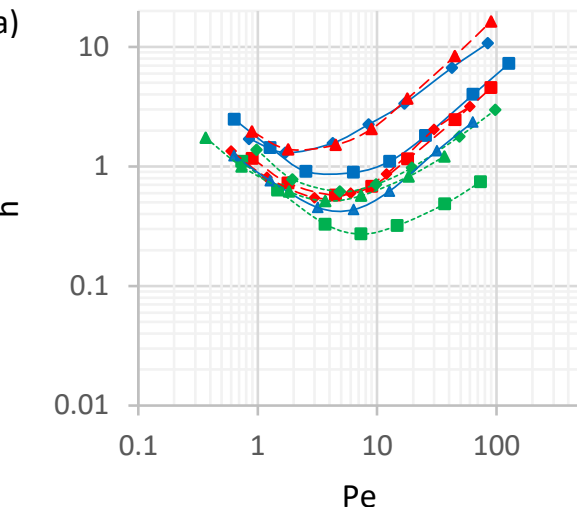

)

$\mathrm{Pe}$

c)

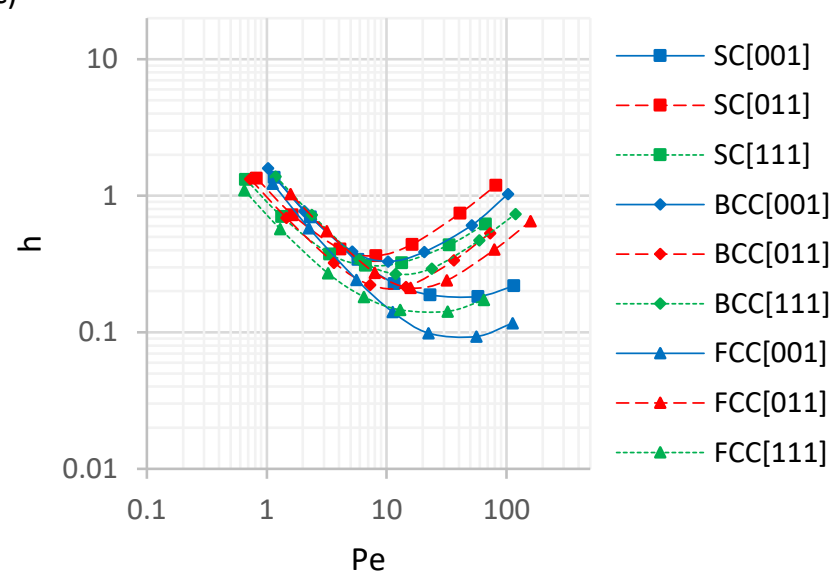

b)

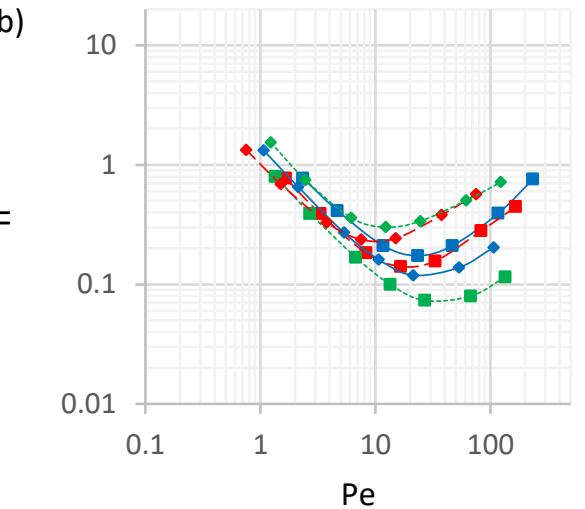

Figure S4. van Deemter curves for ordered packings of a) spherical, b) octahedral and c) tetrahedral particles. For all data in these figures, rotation and $\lambda=1$. The key is applicable for each figure, however, the data set for octahedra does not include the FCC arrangement, because this is a not a permeable packed bed. This figure is supplementary to Fig. 2 in the main text. 

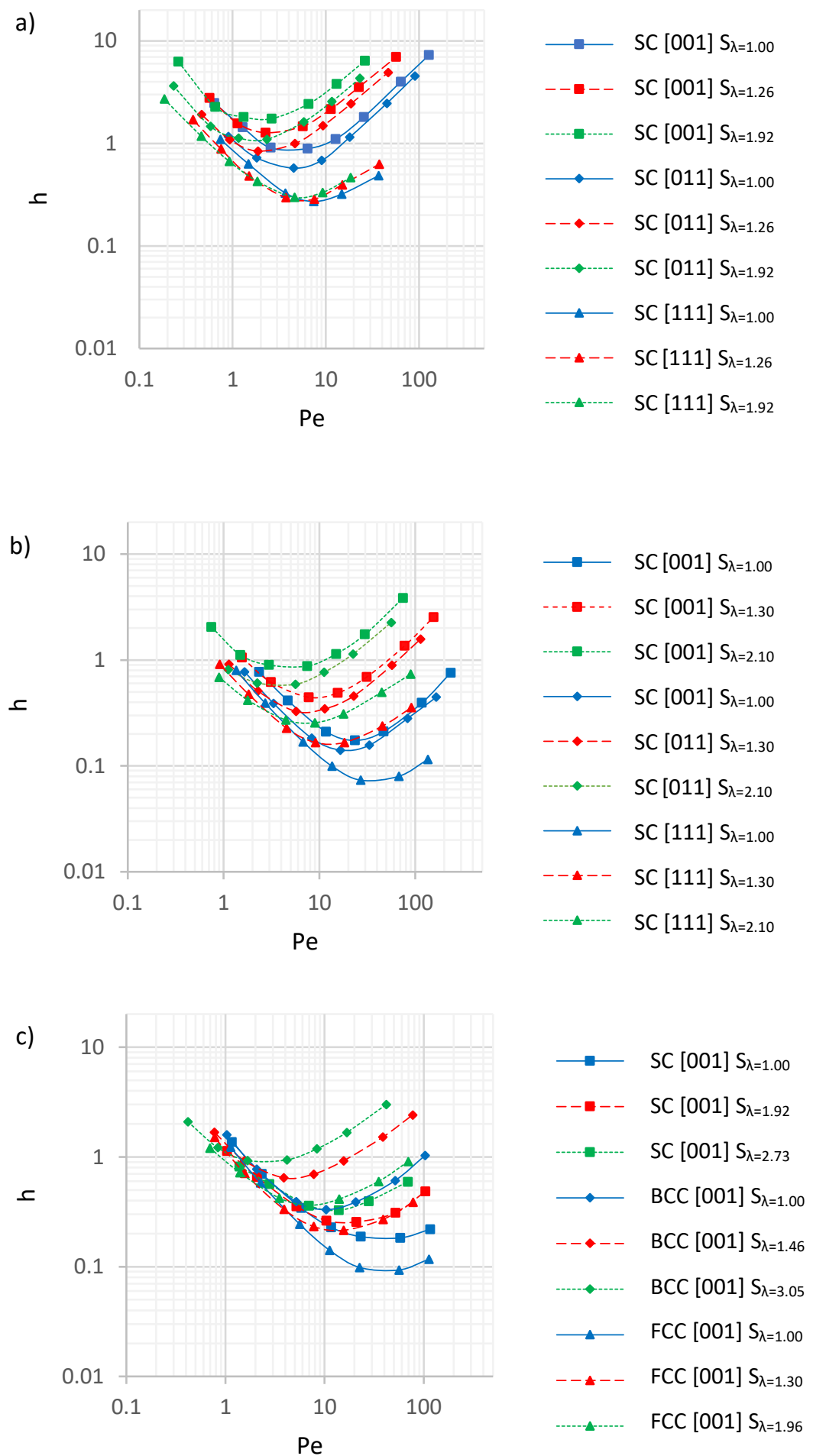

Figure S5. van Deemter curves for overlapped: a) spheres, b) octahedra and c) tetrahedra. This figure is supplementary to Fig. 4 in the main text. 


\section{References}

(1) De Malsche, W.; Eghbali, H.; Clicq, D.; Vangelooven, J.; Gardeniers, H.; Desmet, G. Anal. Chem. 2007, 79, 5915-5926.

(2) Guiochon, G. J. Chromatogr. A 2007, 1168, 101-168.

(3) Dolamore, F.; Fee, C.; Dimartino, S. J. Chromatogr. A 2018, 1532, 150-160.

(4) Khirevich, S.; Höltzel, A.; Seidel-Morgenstern, A.; Tallarek, U. J. Chromatogr. A 2012, 1262, 77-91.

(5) Schure, M. R.; Maier, R. S.; Kroll, D. M.; Davis, H. T. Anal. Chem. 2002, 74, 6006-6016.

(6) Schure, M. R.; Maier, R. S.; Kroll, D. M.; Ted Davis, H. J. Chromatogr. A 2004, 1031, 79-86.

(7) Schure, M. R.; Maier, R. S. J. Chromatogr. A 2018, 1580, 30-48.

(8) Poole, C. F. The Essence of Chromatography; Elsevier, 2003.

(9) Daneyko, A.; Hlushkou, D.; Khirevich, S.; Tallarek, U. J. Chromatogr. A 2012, 1257, 98-115.

(10) Kolev, N. Packed Bed Columns: For Absorption, Desorption, Rectification and Direct Heat Transfer; Elsevier Science, 2006.

(11) Li, L.; Yan, X.; Yang, J.; Wang, Q. Appl. Energy 2017, 185, 2168-2180.

(12) Bird, R. B.; Stewart, W. E.; Lightfoot, E. N. Transport Phenomena; Wiley, 2007. 